\title{
Traumatic Fractures Should Trigger Osteoporosis Assessment in Postmenopausal Women
}

\author{
Crandall CJ, Larson JC, LaCroix AZ, et al. Risk of Subsequent Fractures in Postmenopausal Women \\ After Nontraumatic vs Traumatic Fractures. JAMA Intern Med. Published online June 07, 2021. \\ doi:10.1001/jamainternmed.2021.2617
}

\section{Study Overview}

Objective. To compare the risk of subsequent fractures after an initial traumatic or nontraumatic fracture in postmenopausal women.

Design. A prospective observational study utilizing data from the Women's Health Initiative (WHI) Study, WHI Clinical Trials (WHI-CT), and WHI Bone Density Substudy to evaluate rates at which patients who suffered a traumatic fracture vs nontraumatic fracture develop a subsequent fracture.

Setting and participants. The WHI study, implemented at 40 United States clinical sites, enrolled 161808 postmenopausal women aged 50 to 79 years at baseline between 1993 and 1998. The study cohort consisted of 75335 patients who had self-reported fractures from September 1994 to December 1998 that were confirmed by the WHI Bone Density Substudy and WHI-CT. Of these participants, $253(0.3 \%)$ were excluded because of a lack of follow-up information regarding incident fractures, and 8208 (10.9\%) were excluded due to incomplete information on covariates, thus resulting in an analytic sample of 66874 (88.8\%) participants. Prospective fracture ascertainment with participants was conducted at least annually and the mechanism of fracture was assessed to differentiate traumatic vs nontraumatic incident fractures. Traumatic fractures were defined as fractures caused by motor vehicle collisions, falls from a height, falls downstairs, or sports injury. Nontraumatic fractures were defined as fractures caused by a trip and fall.

Main outcome measures. The primary outcome was an incident fracture at an anatomically distinct body part. Fractures were classified as upper extremity (carpal, elbow, lower or upper end of humerus, shaft of humerus, upper radius/ulna, or radius/ulna), lower extremity (ankle, hip, patella, pelvis, shaft of femur, tibia/fibula, or tibial plateau), or spine (lumbar and/or thoracic spine). Selfreported fractures were verified via medical chart review by WHI study physicians; hip fractures were confirmed by review of written reports of radiographic studies; and nonhip fractures were confirmed by review of radiography reports or clinical documentations.

Main results. In total, 66874 women in the study (mean [SD] age) 63.1 (7.0) years without clinical fracture and 65.3 (7.2) years with clinical fracture at baseline were followed for 8.1 (1.6) years. Of these participants, 7142 (10.7\%) experienced incident fracture during the study follow-up period (13.9

\section{Outcomes Research in Review SECTION EDITORS}

KatRina F. MATEO, PHD, MPH

CUNY School of Public Health New York, NY
DANIEL ISAAC, DO, MS

Michigan State University East Lansing, Ml
FRED Ko, MD, MS

Icahn School of Medicine at Mount Sinai New York, NY
TAISHI HIRAI, MD

University of Missouri

Columbia, $\mathrm{MO}$
William Hung, MD, MPH

Icahn School of Medicine at Mount Sinai

New York, NY 


\section{Outcomes Research in Review}

per 1000 person-years), and 721 (10.1\%) of whom had a subsequent fracture. The adjusted hazard ratio (aHR) of subsequent fracture after an initial fracture was 1.49 (95\% Cl, 1.38-1.61, $P<.001)$. Covariates adjusted were age, race, ethnicity, body mass index, treated diabetes, frequency of falls in the previous year, and physical function and activity. In women with initial traumatic fracture, the association between initial and subsequent fracture was increased (aHR, 1.25; 95\% Cl, 1.06-1.48, $P=.01$ ). Among women with initial nontraumatic fracture, the association between initial and subsequent fracture was also increased (aHR, 1.52; 95\% Cl, 1.37-1.68, $P<.001$ ). The confidence intervals for the 2 preceding associations for traumatic and nontraumatic initial fracture strata were overlapping.

Conclusion. Fractures, regardless of mechanism of injury, are similarly associated with an increased risk of subsequent fractures in postmenopausal women aged 50 years and older. Findings from this study provide evidence to support reevaluation of current clinical guidelines to include traumatic fracture as a trigger for osteoporosis screening.

\section{Commentary}

Osteoporosis is one of the most common age-associated disease that affects 1 in 4 women and 1 in 20 men over the age of $65 .^{1}$ It increases the risk of fracture, and its clinical sequelae include reduced mobility, health decline, and increased all-cause mortality. The high prevalence of osteoporosis poses a clinical challenge as the global population continues to age. Pharmacological treatments such as bisphosphonates are highly effective in preventing or slowing bone mineral density (BMD) loss and reducing risk of fragility fractures (eg, nontraumatic fractures of the vertebra, hip, and femur) and are commonly used to mitigate adverse effects of degenerative bone changes secondary to osteoporosis. ${ }^{1}$

The high prevalence of osteoporosis and effectiveness of bisphosphonates raises the question of how to optimally identify adults at risk for osteoporosis so that pharmacologic therapy can be promptly initiated to prevent disease progression. Multiple osteoporosis screening guidelines, including those from the
United States Preventive Services Task Force (USPSTF), American Association of Family Physicians, and National Osteoporosis Foundation, are widely used in the clinical setting to address this important clinical question. In general, the prevailing wisdom is to screen osteoporosis in postmenopausal women over the age of 65 , women under the age of 65 who have a significant 10-year fracture risk, or women over the age of 50 who have experienced a fragility fracture. ${ }^{1}$ In the study reported by Crandall et al, it was shown that the risks of having subsequent fractures were similar after an initial traumatic or nontraumatic (fragility) fracture in postmenopausal women aged 50 years and older. ${ }^{2}$ This finding brings into question whether traumatic fractures should be viewed any differently than nontraumatic fractures in women over the age of 50 in light of evaluation for osteoporosis. Furthermore, these results suggest that most fractures in postmenopausal women may indicate decreased bone integrity, thus adding to the rationale that osteoporosis screening needs to be considered and expanded to include postmenopausal women under the age of 65 who endured a traumatic fracture.

Per current guidelines, a woman under the age of 65 is recommended for osteoporosis screening only if she has an increased 10-year fracture risk compared to women aged 65 years and older. This risk is calculated based on the World Health Organization fracture-risk algorithm (WHO FRAX) tool which uses multiple factors such as age, weight, and history of fragility fractures to predict whether an individual is at risk of developing a fracture in the next 10 years. The WHO FRAX tool does not include traumatic fractures in its risk calculation and current clinical guidelines do not account for traumatic fractures as a red flag to initiate osteoporosis screening. Therefore, postmenopausal women under the age of 65 are less likely to be screened for osteoporosis when they experience a traumatic fracture compared to a fragility fracture, despite being at a demonstrably higher risk for subsequent fracture. As an unintended consequence, this may lead to the under diagnosis of osteoporosis in postmenopausal women under the age of 65. Thus, Crandall et al conclude that a fracture due to any cause warrants follow up evaluation for osteoporosis including BMD testing in women older than 50 years of age. 
Older men constitute another population who are commonly under screened for osteoporosis. The current USPSTF guidelines indicate that there is an insufficient body of evidence to screen men for osteoporosis given its lower prevalence. ${ }^{1}$ However, it is important to note that men have significantly increased mortality after a hip fracture, are less likely to be on pharmacological treatment for osteoporosis, and are under diagnosed for osteoporosis. ${ }^{3}$ Consistent with findings from the current study, Leslie et al showed that high-trauma and low-trauma fractures have similarly elevated subsequent fracture risk in both men and women over the age of 40 in a Canadian study. ${ }^{4}$ Moreover, in the same study, BMD was decreased in both men and women who suffered a fracture regardless of the injury mechanism. This finding further underscores a need to consider traumatic fractures as a risk factor for osteoporosis. Taken together, given that men are under screened and treated for osteoporosis but have increased mortality post-fracture, considerations to initiate osteoporosis evaluation should be similarly given to men who endured a traumatic fracture.

The study conducted by Crandall et al has several strengths. It is noteworthy for the large size of the WHI cohort with participants from across the United States which enables the capture of a wider range of age groups as women under the age of 65 are not common participants of osteoporosis studies. Additionally, data ascertainment and outcome adjudication utilizing medical records and physician review assure data quality. A limitation of the study is that the study cohort consists exclusively of women and therefore the findings are not generalizable to men. However, findings from this study echo those from other studies that investigate the rela- tionship between fracture mechanisms and subsequent fracture risk in men and women., Collectively, these comparable findings highlight the need for additional research to validate traumatic fracture as a risk factor for osteoporosis and to incorporate it into clinical guidelines for osteoporosis screening.

\section{Applications for Clinical Practice}

The findings from the current study indicate that traumatic and fragility fractures may be more alike than previously recognized in regards to bone health and subsequent fracture prevention in postmenopausal women. If validated, these results may lead to changes in clinical practice whereby all fractures in postmenopausal women could trigger osteoporosis screening, assessment, and treatment if indicated for the secondary prevention of fractures.

$$
\text { -lan Chun, BS, and Fred Ko, MD }
$$

doi:10.12788/jcom.0057

\section{References}

1. USPreventive Services Task Force, Curry SJ, Krist Ah, et al. Screening for Osteoporosis to Prevent Fractures: US Preventive Services Task Force Recommendation Statement. JAMA. 2018;319(24):25212531. doi:10.1001/jama.2018.7498

2. Crandall CJ, Larson JC, LaCroix AZ, et al. Risk of Subsequent Fractures in Postmenopausal Women After Nontraumatic vs Traumatic Fractures. JAMA Intern Med. Published online June 7, 2021. doi:10.1001/jamainternmed.2021.2617

3. Mackey DC, Lui L, Cawthon PM, et al. High-Trauma Fractures and Low Bone Mineral Density in Older Women and Men. JAMA. 2007;298(20):2381-2388. doi:10.1001/jama.298.20.2381

4. Leslie WD, Schousboe JT, Morin SN, et al. Fracture risk following high-trauma versus low-trauma fracture: a registry-based cohort study. Osteoporos Int. 2020;31(6):1059-1067. doi:10.1007/ s00198-019-05274-2 


\title{
Nivolumab Plus Cabozantinib Improves Outcomes Compared With Sunitinib for Advanced Renal Cell Carcinoma
}

\author{
Choueiri TK, Powles T, Burotto $M$, et al. Nivolumab plus Cabozantinib versus Sunitinib \\ for Advanced Renal-Cell Carcinoma. N Engl J Med. 2021;384(9):829-841. \\ Doi:10.1056/NEJMoa2026982
}

\section{Study Overview}

Objective. To evaluate the efficacy and safety of the combination of nivolumab plus cabozantinib as compared with sunitinib monotherapy in the treatment of previously untreated advanced renal cell carcinoma (RCC).

Design. Multicenter, international, open-label, randomized, phase 3 trial.

Intervention. Patients were randomized in a 1:1 fashion to 1 of 2 treatment arms:

- Arm A: Nivolumab intravenously 240 mg every 2 weeks plus cabozantinib orally $40 \mathrm{mg}$ once daily.

- Arm B: Sunitinib orally 50 mg daily for 4 weeks, followed by 2 weeks off therapy (6-week cycle).

Randomization was stratified by the International Metastatic RCC Database Consortium prognostic risk score (low-, intermediate-, and high-risk). Treatment was continued until disease progression or development of unacceptable toxic side effects with a maximum of 2-year duration of Nivolumab therapy.

Settings and participants. Adults with previously untreated advanced RCC with a clear cell component were eligible for enrollment. Subjects were excluded if they had active central nervous system metastases or active autoimmune disease.

Main outcome measures. The primary outcome of this study was progression-free survival (PFS) as assessed by an independent review committee. Secondary endpoints included overall survival, objective response rate, safety, and PFS as assessed by investigators. All subgroup analyses were prespecified. Efficacy was assessed in the intention-to-treat population, including all patients who underwent randomization.

Main results. A total of 651 patients underwent randomization: 323 to the nivolumab plus cabozantinib group, and 328 to the sunitinib group. Baseline demographics were balanced. The median follow-up period for overall survival (OS) was 18.1 months. The primary reason for treatment discontinuation in any group was disease progression. PFS as indicated by an independent review committee was significantly longer in the nivolumab plus cabozantinib group compared to the sunitinib group (median 16.6 months vs 8.2 months; hazard ratio $[\mathrm{HR}] 0.51, P<.001)$. The median OS was not reached for any group. Overall survival was longer in the nivolumab plus cabozantinib group compared to the sunitinib group ( $\mathrm{HR} 0.60,95 \% \mathrm{Cl}$ : 0.40-0.89; $P=.001$ ). The objective response rate was $55.7 \%$ with the nivolumab plus cabozantinib group versus $27.1 \%$ with sunitinib $(P<.001)$. The complete response rate was $8 \%$ in the nivolumab plus cabozantinib group compared to $4.6 \%$ in the sunitinib group. The median time to response was 2.8 months with nivolumab plus cabozantinib and 4.2 months in the sunitinib group, while the median duration of response was 20.2 months and 11.5 months, respectively.

Nearly all patients (about 99\% in each group) had an adverse event (AE). Hypertension was the most common side effect, with grade 3 or higher seen in $12.5 \%$ in the nivolumab plus cabzantinib group and $13.1 \%$ in the sunitinib group. Other grade 3 or higher side effects occurring in at least $10 \%$ of patients in any group were hyponatremia, diarrhea, palmar-plantar erythrodysesthesia, hypothyroidism, and fatigue. AEs 


\section{Outcomes Research in Review}

of any cause leading to discontinuation of the therapy occurred in $19.7 \%$ in the nivolumab plus cabzantinib group vs $16.9 \%$ of the sunitinib group. One death was considered to be treatment-related (small intestinal perforation) in the nivolumab plus cabozantinib group vs 2 treatment-related deaths with sunitinib (pneumonia and respiratory distress). In the nivolumab plus cabozantinib group, $57 \%$ of the patients had a dose reduction of cabozantinib and $52 \%$ had a reduction in sunitinib dosage.

Using the Functional Assessment of Cancer TherapyKidney Symptoms Index, patients in the nivolumab plus cabozantinib group reported better health-related quality of life and less disease-related symptoms compared to the sunitinib group.

\section{Commentary}

The treatment landscape for frontline therapy for patients with advanced RCC has rapidly expanded over the last several years and has revolutionized cancer care. Ushered in by the results from the CheckMate 214 study highlighting the efficacy of dual checkpoint inhibition with nivolumab and ipilimumab in intermediate and poor risk patients, several subsequent trials have demonstrated improved outcomes with combination therapy with immune checkpoint inhibitors and tyrosine-kinase inhibitors (TKI). To date, data from Keynote-426 (pembrolizumab plus axitinib vs sunitinib), Javelin Renal 101 (avelumab plus axitinib vs sunitinib) and the CLEAR trial (lenvatinib plus pembrolizumab vs levatinib plus everolimus vs sunitinib) have demonstrated superiority of immune checkpoint inhibitor/TKI combinations over sunitinb in the first-line setting. ${ }^{1-5}$

The current phase 3, CheckMate 9ER trial adds yet another dynamic option for patients with advanced clear cell RCC. While cross-trial comparisons are fraught with important caveats, the median PFS of almost 16.6 months and complete response rate of $8 \%$ the nivolumab plus cabozantinib group compares favorably with other combinations. Data from the CLEAR study with the combination of lenvatinib and pembrolizumab showed a complete response rate approaching $16 \%$. Importantly, the current study highlights improved quality of life with the combination of cabozantinib and nivolumab compared to sunitinib alone adding to the efficacy and benefits of this combination treatment.

The selection of first line therapy for patients with advanced RCC should be always guided by individual patient characteristics, and any single immune checkpoint inhibitor/TKI combination is not "superior" to any other. Perhaps more importantly is developing an understanding of the overlapping toxicity profiles of checkpoint inhibitors and TKIs. Again, this trial results are consistent with prior studies in terms of the adverse event profile which were not trivial, and almost all patients (99\%) experienced AEs. It is important for oncologists to understand the management of the toxicities with these combinations and dose reductions as appropriate. It is worth noting that $19 \%$ of patients with nivolumab plus cabozantinib received glucocorticoids for management of immune-related AEs.

While long-term follow-up data will be needed to further understand the durability of response to this combination, nivolumab-cabozantinib represents an exciting new option for patients with advanced clear cell RCC. As we continue to see improvement in outcomes in clear cell histology, further work must focus on optimization of therapy in non-clear cell RCC as this is a population that is not represented in these data sets. Furthermore, future efforts should begin to explore triplet combinations and biomarker driven patient selection for upfront therapy in order continue to improve outcomes in patients with advanced RCC.

\section{Applications for Clinical Practice}

The combination of nivolumab plus cabozantinib adds to the growing list of highly active checkpoint inhibitor/TKI combinations for first-line treatment of advanced RCC. With significant higher response rates, improved outcomes, and improvement in the quality of life, this combination will add another standard treatment option for patients with previously untreated advanced RCC.

-Daniel Isaac, DO, MS, Osama Mosalem, MD dol:10.12788/jcom.0058 


\section{References}

1. Motzer RJ, Tannir NM, McDermott DF, et al. Nivolumab plus Ipilimumab Versus Sunitinib in Advanced Renal-Cell Carcinoma. $N$ Engl J Med. 2018;378(14)1277-1290. doi:10.1056/ NEJMoa1712126

2. Rini Bl, Plimack ER, Stus V, et al. Pembrolizumab plus Axitinib versus Sunitinib for Advanced Renal-Cell Carcinoma. N Engl J Med. 2019;380(12):1116-1127. doi:10.1056/NEJMoa1816714

3. Powles T, Plimack ER, Soulières D, et al. Pembrolizumab plus axitinib versus sunitinib monotherapy as first-line treatment of advanced renal cell carcinoma (KEYNOTE-426): extended follow- up from a randomised, open-label, phase 3 trial. Lancet Oncol. 2020;21(12):1563-1573. doi:10.1016/S1470-2045(20)30436-8

4. Choueiri TK, Motzer RJ, Rini Bl, et al. Updated efficacy results from the JAVELIN Renal 101 trial: first-line avelumab plus axitinib versus sunitinib in patients with advanced renal cell carcinoma. Ann Oncol. 2020;31:1030-1039. doi:10.1016/j.annonc.2020. 04.010

5. Motzer R, Alekseev B, Rha SY, et al. CLEAR Trial Investigators. Lenvatinib plus Pembrolizumab or Everolimus for Advanced Renal Cell Carcinoma. N Engl J Med. 2021;384(14):1289-1300. doi:10.1056/NEJMoa2035716 